\title{
Exact Solutions in a Model of Vertical Gas Migration
}

\author{
Dmitriy B. Silin, SPE, LBNL, UC Berkeley \\ Tad W. Patzek, SPE, UC Berkeley, LBNL \\ Sally M. Benson, SPE, $L B N L$
}

The analytical results are applied to studying carbon dioxide flow caused by leaks from deep geological formations used for $\mathrm{CO}_{2}$ storage. The results are also applicable for modeling flow of natural gas leaking from seasonal gas storage, or for modeling of secondary hydrocarbon migration.

\section{Introduction}

This work is motivated by the growing interest in injecting carbon dioxide into deep geological formations as a means of avoiding atmospheric emissions of carbon dioxide and consequent global warming. ${ }^{1}$ One of the key questions regarding the feasibility of this technology is the potential rate of leakage out of the primary storage formation. To date, studies of leakage have either focused on the role of leaking wells ${ }^{2,3}$ or used numerical simulation to investigate leakage through poor-quality reservoir seals., ${ }^{4} 5$ Here we are interested in the fate of $\mathrm{CO}_{2}$ driven upwards by buoyancy forces, using analytical methods to help provide insight into the physics of countercurrent flow of $\mathrm{CO}_{2}$ and water and to bound potential migration rates.

In steady-state flow, two immiscible fluids saturating a porous medium approach an equilibrium distribution characterized by a local energy minimum. In this study, we focus on a case where a less dense fluid, e.g., gas, migrates upward in an initially dense-liquid-saturated reservoir. The driving forces in this flow are the buoyancy resulting from the contrast between the fluid densities, and the capillary forces acting at the interfaces between the fluids and solid. The character of rock wettability impacts the capillary pressure and relative permeability curves. Although capillary forces act locally at a microscopic scale, they constrain the macroscopic flow by arranging the distribution of the fluids. Examples of such flows are oil and gas migration, ${ }^{6,7}$ or the flow of gas leaking from an underground storage into an overlying aquifer. Shvidler and Levi $^{8}$ obtained an analytical solution to a static gravity segregation problem and numerically investigated dynamic gravity-driven flow in 1D. Hydrocarbon migration prob- 


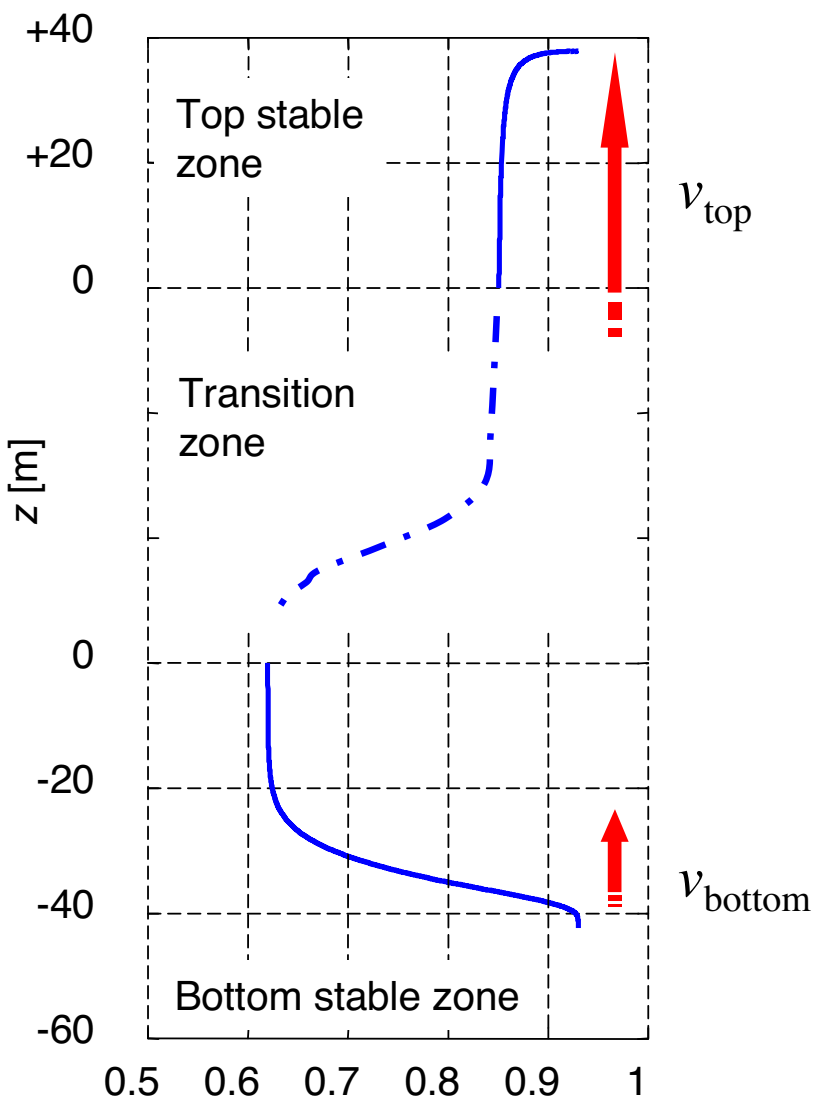

$\mathrm{S}$

Fig. 1 - The top part of the plume is relatively "lean" with respect to the gas saturation, but propagates much faster than the bottom part saturated with gas.

lems have been studied recently in the context of geologic formation of oil and gas reservoirs. ${ }^{9,10}$ An analytical solution for a model of gas plume propagation in a saline aquifer caused by gas injection has been obtained by Nordbotten et al. ${ }^{3}$ Their solution is based on the BuckleyLeverett model that neglects the capillarity. Doughty has investigated numerically the impact of capillary hysteresis effects on $\mathrm{CO}_{2}$ migration. ${ }^{11}$

In this study, we analyze the buoyancy-driven vertical gas plume migration using analytical tools. Our main finding is that a moving plume has two stable zones: at the top and at the bottom. We obtain an estimate of the theoretical maximum of the plume migration velocity, which strongly depends on the relative permeability curves. Each one of the stable parts of the plume is characterized by a travelling-wave solution. It turns out, that the top part of the plume propagates with the maximal velocity, whereas the bottom one moves more slowly, Fig. 1.

We assume that the overlying formation is homogeneous. This assumption is an idealization. Fluid migra- tion is significantly affected by the rock heterogeneity. For instance, if the formation into which the gas leaks has a system of vertical or inclined conductive fractures or faults, the gas, most likely, will flow through this system.

In our model, gas is the nonwetting fluid, whereas the rock is wetted by the liquid brine, which initially saturates the pore space. At first, the high-pressure gas will drain the liquid from the pore space at the bottom part of the aquifer. With time, this gas is buoyed by the denser brine and flows upward. Since the capillary entry pressure for the small pores and the corners between the rock grains can be extremely high, appearance of absolutely dry zones without evaporation is unlikely. We thus neglect processes like liquid circulation due to the evaporation and condensation. Therefore, it is assumed that the pore space is partially saturated with both fluids and they flow in their respective flow paths determined by the equilibrium of the interfacial forces and gravity. The non-wetting gas will flow through the central parts of the pores, whereas the water will flow through the small pores, corners and roughness of the solid skeleton. While flowing upward into the portions of the less compressed reservoir layers, the gas expands.

In every elementary representative volume, or, at each point in our model, the fluids have different pressures due to capillarity. The difference between the gas and liquid pressures, the capillary pressure, determines the mean radii of curvature of the gas-liquid interfaces in the pore space. In this study, we neglect the dependence of the surface tension coefficient on the temperature and interfacial impurities. Thermal effects, as well as the impact of water transport by evaporation will be considered elsewhere.

Countercurrent two-phase flow in porous media has been intensely studied, both experimentally and theoretically, in the context of enhanced oil recovery. ${ }^{12,13}$ The combination of natural time and length scales in such studies suggest that the compressibility of the fluids can be neglected. In case of gas migration, such an assumption is questionable, especially when dealing with shallow depths. In this study, we assume that the domain under consideration is deep enough so that the gas is supercritical and the variation of density over the interval of depths of interest is negligibly small. In other words, this study focuses on the model of gas plume migration resulting from the interaction between gravity, capillary, and viscous forces only. The paper is organized as follows. First, we briefly review the gravity-segregation model and formulate the main equations of countercurrent flow of gas and brine. Penetration by a gas plume of a low-permeability seal is studied using a Ryzhik-type model, neglecting gravity forces. A more general approach is applied to plume vertical migration in an aquifer, where two travelling-wave solutions describe two stable zones at the top and at the bottom of the plume. Conclusions and the nomenclature are given at the end. The parameters used in the case studies presented in this paper are gathered in Appendix. 


\section{The model}

Consider buoyancy-driven flow of gas in a vertical fracture or a thick horizontal aquifer. The medium is filled with brine, which is the wetting fluid. We neglect the lateral flow components, so the flow is essentially vertical. This assumption is valid if the gas flow is horizontally confined by the fracture walls or if we consider migration of gas in an aquifer far away from the lateral boundaries of the plume. Therefore, the flow is two-phase and countercurrent: the gas flowing upward is replaced with an equal volume of the brine flowing downward. In this section, we review the principle equations of the mathematical model.

Denote by $p_{g}$ and $p_{w}$ the gas and brine pressures, respectively. At each point, the capillary pressure, $p_{c}=$ $p_{g}-p_{w}$, is related to the distribution of the fluids in the the pore space, whose microscopic-scale geometry is usually extremely complex. At the macroscopic scale, this distribution is quantified by the relative volume of the pore space filled with the liquid, $S$. Thus, the capillary pressure is a function of $S$ :

$$
p_{g}-p_{w}=p_{c}(S)
$$

This dependence on $S$ is not a one-to-one correspondence, ${ }^{7}$ but is strongly affected by the history of fluid migration. Numerous studies emphasize the difference between drainage and imbibition capillary pressure curves, see $e^{14,15}$ and the references therein. The capillary pressure - water saturation relationship behind the rising gas plume, where the displaced gas is again replaced by the wetting liquid (secondary imbibition), is different from that at the top of the plume.

Since each fluid fills only a part of the pore space, the permeability to a fluid is determined by the geometry of the fluid distribution in the pores. Inasmuch as the fluid distribution is not uniquely determined by water saturation, the permeability to each fluid is a function of a combination of the liquid saturation and the history of the fluid migration. Hence, the remarks regarding the historydependence of the capillary pressure curve equally apply to the relative permeability curves. Again, since we study gas invasion into an aquifer, we use the drainage relative permeability factors $k_{r g}=k_{r g}(S)$ and $k_{r w}=k_{r w}(S)$. Thus, if the absolute permeability of the formation is $k$, then the permeability to the gas is $k_{r g}(S) k$, whereas that to the liquid is $k_{r w}(S) k$.

Darcy's law with an account for gravity, ${ }^{16}$ applied to each fluid phase separately, yields:

$$
\begin{aligned}
\mathbf{u}_{g} & =\frac{k_{r g}(S) k}{\mu_{g}}\left(-\nabla p_{g}+\varrho_{g} \mathbf{g}\right) \\
\mathbf{u}_{w} & =\frac{k_{r w}(S) k}{\mu_{w}}\left(-\nabla p_{w}+\varrho_{w} \mathbf{g}\right)
\end{aligned}
$$

Here $\mathbf{u}_{g}$ and $\mathbf{u}_{w}$ are, respectively, Darcy velocities, or volumetric fluxes, of the gas and liquid, $\mu_{g}$ and $\mu_{w}$ are the dynamic viscosities of the fluids, $\varrho_{g}$ and $\varrho_{w}$ are their densities. The gravity acceleration is denoted by $\mathbf{g}$. The fluid does not flow, $\mathbf{u}=0$, if the pressure gradient is equilibrated by the gravity term: $\nabla p_{i}=\varrho_{i} \mathbf{g}, i=g, w$. Thus, if $z$ is the vertical axis with the positive direction upward, then the equilibrium conditions read

$$
\begin{aligned}
p_{w}(z) & =p_{w}\left(z_{0}\right)-\varrho_{w} g\left(z-z_{0}\right) \\
p_{g}(z) & =p_{g}\left(z_{0}\right)-\varrho_{g} g\left(z-z_{0}\right)
\end{aligned}
$$

Here $g$ is the scalar magnitude of the gravity acceleration.

The mass balance of the gas and liquid can be expressed as

$$
\begin{aligned}
\frac{\partial\left(\varrho_{g}(1-S) \phi\right)}{\partial t}+\nabla \cdot\left(\varrho_{g} \mathbf{u}_{g}\right) & =0 \\
\frac{\partial\left(\varrho_{w} \phi S\right)}{\partial t}+\nabla \cdot\left(\varrho_{w} \mathbf{u}_{w}\right) & =0
\end{aligned}
$$

Equations (1)-(6) constitute a system of equations with seven unknown functions: $\mathbf{u}_{i}, p_{i}, \varrho_{i}(i=g, w)$, and $S$. In fact, Darcy velocities are vector quantities. Therefore, each of the equations (2)-(3) is a system of three scalar equations, and the number of unknown functions is equal to eleven. A unique solution to the system (1)-(6) can be determined if it is complemented with the equations of state for both fluids, and with a consistent set of boundary and initial conditions.

If the compressibility of the gas, the brine and the rock can be neglected, summation of Equations (5)-(6) yields

$$
\nabla \cdot\left(\mathbf{u}_{g}+\mathbf{u}_{w}\right)=0
$$

In general, Equation (7) does not imply yet that the total Darcy velocity of both fluids is constant. The latter holds true if, in addition to (7),

$$
\nabla \times\left(\mathbf{u}_{g}+\mathbf{u}_{w}\right)=0
$$

which is not necessarily true. However, if the flow is onedimensional, for example after averaging in the two horizontal coordinates, (7) reduces to a single scalar equation

$$
u_{g}+u_{w}=\text { Const }
$$

If the constant in the last equation is equal to zero, the flow is countercurrent. ${ }^{17-19}$ In this work, we consider vertical migration of a gas plume with no fluid flow at a large distance from the plume. Hence, in this case,

$$
u_{g}+u_{w}=0
$$

Using capillary pressure equation (1), the liquid pressure $p_{w}$ can be eliminated from the system of equations (1)-(6). Thus, from Equation (1), and Darcy's law for the brine (3) can be written down in the following way:

$$
\mathbf{u}_{w}=\frac{k_{r w}(S) k}{\mu_{w}}\left(-\nabla p_{g}+\nabla p_{c}(S)+\varrho_{w} \mathbf{g}\right)
$$




\section{Countercurrent flow}

In this section, the vertical transient migration of a gas plume is studied. We assume that the flow is countercurrent, i.e., Equation (10) holds true. The countercurrent flow equation obtained in this Section is similar to the one obtained by Luan. ${ }^{20}$ The governing equations are nondimensionalized to a form similar to the Rappoport-Leas water-oil displacement equation. ${ }^{21}$ This analogy suggests two asymptotic forms. In the first case, the gas propagates through a relatively thin low-permeability seal. Here, capillary forces dominate over gravity and the process is characterized by a self-similar solution similar to Ryzhik's model of spontaneous imbibition. ${ }^{18}$ In the second case, the flow is considered in a relatively thick aquifer, so that gravity forces cannot be neglected. In this case, two stable zones at the top and at the bottom of the plume are described through two different travelling-wave solutions.

In the case of countercurrent flow of gas migrating upwards and brine flowing downwards, the total Darcy velocity of the mixture is equal to zero, equation (10). Therefore, Equations (2) and (11) imply

$$
\begin{aligned}
& \frac{\partial}{\partial z} p_{g}=\frac{1}{\frac{k_{r w}(S)}{\mu_{w}}+\frac{k_{r g}(S)}{\mu_{g}}} \\
& \times\left(\frac{k_{r w}(S)}{\mu_{w}} \frac{\partial}{\partial z} p_{c}(S)-\frac{k_{r g}(S)}{\mu_{g}} \varrho_{g} g-\frac{k_{r w}(S)}{\mu_{w}} \varrho_{w} g\right)
\end{aligned}
$$

For the water pressure gradient, Equation (1) implies

$$
\begin{aligned}
& \frac{\partial}{\partial z} p_{w}=-\frac{1}{\frac{k_{r w}(S)}{\mu_{w}}+\frac{k_{r g}(S)}{\mu_{g}}} \\
& \times\left(\frac{k_{r g}(S)}{\mu_{g}} \frac{\partial}{\partial z} p_{c}(S)+\frac{k_{r g}(S)}{\mu_{g}} \varrho_{g} g+\frac{k_{r w}(S)}{\mu_{w}} \varrho_{w} g\right)
\end{aligned}
$$

Equivalently, equations (12)-(13) can be written down as

$$
\frac{\partial}{\partial z} p_{g}=\frac{\frac{k_{r w}(S)}{\mu_{w}}}{\frac{k_{r w}(S)}{\mu_{w}}+\frac{k_{r g}(S)}{\mu_{g}}}\left[\frac{\partial}{\partial z} p_{c}(S)-\left(\varrho_{w}-\varrho_{g}\right) g\right]-\varrho_{g} g
$$

and

$$
\frac{\partial}{\partial z} p_{w}=-\frac{\frac{k_{r g}(S)}{\mu_{g}}}{\frac{k_{r w}(S)}{\mu_{w}}+\frac{k_{r g}(S)}{\mu_{g}}}\left[\frac{\partial}{\partial z} p_{c}(S)-\left(\varrho_{w}-\varrho_{g}\right) g\right]-\varrho_{w} g
$$

By virtue of equation (15), the Darcy velocity of water is

$$
u_{w}=\frac{k}{\mu_{w}} f(S)\left[\frac{\partial}{\partial z} p_{c}(S)-\left(\varrho_{w}-\varrho_{g}\right) g\right]
$$

where

$$
f(S)=\frac{k_{r w}(S) \frac{k_{r g}(S)}{\mu_{g}}}{\frac{k_{r w}(S)}{\mu_{w}}+\frac{k_{r g}(S)}{\mu_{g}}}=\frac{k_{r w}(S)}{\frac{k_{r w}(S)}{k_{r g}(S)} \frac{\mu_{g}}{\mu_{w}}+1}
$$

In equation (16), we have left $\mu_{w}$ outside the function $f(S)$ to make the latter dimensionless. There is no flow if

$$
\frac{\partial S}{\partial z}=\frac{\left(\varrho_{w}-\varrho_{g}\right) g}{p_{c}^{\prime}(S)}
$$

Since $p_{c}^{\prime}(S)<0$, this is possible only if $\frac{\partial S}{\partial z}<0$. Equation (18) can be integrated:

$$
\left(z-z_{0}\right)\left(\varrho_{w}-\varrho_{g}\right) g=p_{c}(S(z))-p_{c}\left(S\left(z_{0}\right)\right)
$$

Thus, equilibrium water saturation distribution is provided by the capillary pressure curve.

In most cases of interest, the viscosity of gas is much smaller than that of water. For intermediate saturations, where the relative permeability to gas is appreciably different from zero, the contrast between gas and brine viscosities makes possible to put

$$
f(S) \approx k_{r w}(S)
$$

By virtue of Equation (16) with this approximation, the dimensional Darcy velocity of water is equal to

$$
u_{w}=\frac{k k_{r w}(S)}{\mu_{w}}\left[\frac{\partial}{\partial z} p_{c}(S)-\left(\varrho_{w}-\varrho_{g}\right) g\right]
$$

If the relative permeability to gas is so small that $\frac{k_{r w}(S)}{\mu_{w}} \gg \frac{k_{r g}(S)}{\mu_{g}}$, then

$$
f(S) \approx \frac{\mu_{w}}{\mu_{g}} k_{r g}(S)
$$

In this case,

$$
u_{w}=\frac{k k_{r g}(S)}{\mu_{g}}\left[\frac{\partial}{\partial z} p_{c}(S)-\left(\varrho_{w}-\varrho_{g}\right) g\right]
$$

In other words, normally, the two-phase flow is determined by the flow of water, whereas at extremely low gas saturation, the flow is controlled by gas.

Substitution of Equation (16) into Equation (6) yields

$$
\frac{\partial(\phi S)}{\partial t}=-\frac{\partial}{\partial z}\left(\frac{k}{\mu_{w}} f(S)\left(\frac{\partial}{\partial z} p_{c}(S)-\left(\varrho_{w}-\varrho_{g}\right) g\right)\right)
$$

Let $H$ denote the thickness of the aquifer. Then, a dimensionless time $\tau$ and coordinate $\zeta$ can be introduced in the following way:

$$
\zeta=\frac{z}{H} \quad \text { and } \quad \tau=\frac{k\left(\varrho_{w}-\varrho_{g}^{*}\right) g}{\mu_{w} H} t
$$

Here $\varrho_{g}^{*}$ is the mean value of the gas density. The dimensionless Darcy velocity of water $W_{w}$ has the following form:

$$
\begin{aligned}
& W_{w}=\frac{\mu_{w}}{k\left(\varrho_{w}-\varrho_{g}^{*}\right) g} u_{w} \\
& =f(S)\left[\frac{1}{\left(\varrho_{w}-\varrho_{g}^{*}\right) g} \frac{\partial}{\partial z} p_{c}(S)-\frac{\varrho_{w}-\varrho_{g}}{\varrho_{w}-\varrho_{g}^{*}}\right]
\end{aligned}
$$


At a sufficiently large depth, the gas density variation is significantly smaller than the density contrast between gas and water:

$$
\frac{\varrho_{g}^{*}-\varrho_{g}}{\varrho_{w}-\varrho_{g}^{*}} \ll 1
$$

Thus,

$$
\frac{\varrho_{w}-\varrho_{g}}{\varrho_{w}-\varrho_{g}^{*}}=1+\frac{\varrho_{g}^{*}-\varrho_{g}}{\varrho_{w}-\varrho_{g}^{*}} \approx 1
$$

The capillary pressure function can be expressed through the dimensionless Leverett's $\mathcal{J}$-function: ${ }^{22}$

$$
p_{c}(S)=\sigma \sqrt{\frac{\phi}{k}} \mathcal{J}(S)
$$

where $\sigma$ is the surface tension coefficient at the water-gas interface. Thus, Equations (24) and (26) take on the form

$$
\phi \frac{\partial S}{\partial \tau}=-\frac{\partial}{\partial \zeta} W_{w}
$$

and

$$
W_{w}=f(S)\left(\gamma \mathcal{J}^{\prime}(S) \frac{\partial S}{\partial \zeta}-1\right)
$$

where

$$
\gamma=\frac{\sigma}{\left(\varrho_{w}-\varrho_{g}^{*}\right) g H} \sqrt{\frac{\phi}{k}}
$$

The dimensionless factor $\gamma$ evaluates the ratio between capillary and buoyancy forces. Its value is of the order of 1 for carbon dioxide flowing in a 20 meters thick layer of permeability of the order of 100 millidarcy and porosity about 20 percent at a depth of several kilometers. However, the magnitude of $\gamma$ is much larger in a thin low-permeability seal. For instance, if the permeability is of the order of 0.01 millidarcy, the porosity is around $1 \%$ and the thickness is of the order of 1 meter, then $\gamma \sim 10^{3}$, which is three orders of magnitude larger than in an aquifer.

With such parameters of the fluids and porous medium, a unit interval for the dimensionless time $\tau$ in an aquifer roughly corresponds to 6 weeks, whereas for a tight seal the respective time scale is of the order of hundreds of thousands of years.

Equations (30)-(31) can be summarized in the form of a nonlinear diffusion-advection equation

$$
\phi \frac{\partial S}{\partial \tau}=\frac{\partial}{\partial \zeta}\left[f(S)\left(-\gamma \mathcal{J}^{\prime}(S) \frac{\partial S}{\partial \zeta}+1\right)\right]
$$

If the vertical dimension of the gas plume is large, then, away from its top and bottom, where the saturation variation is not large, the first term on the right-hand side of Equation (33) is much smaller than one:

$$
\left|\gamma \mathcal{J}^{\prime}(S) \frac{\partial S}{\partial \zeta}\right| \ll 1
$$

Since inside the plume the relative permeability to gas is appreciably positive, the approximate expression (20) is valid. In this case, Equation (33) reduces to

$$
\phi \frac{\partial S}{\partial \tau}-\frac{\partial k_{r w}(S)}{\partial \zeta}=0
$$

By integration in $\zeta$, Equation (35) transforms into a Hamilton-Jacobi equation

$$
\frac{\partial U}{\partial t}=k_{r w}\left(\frac{\partial U}{\partial \zeta}\right)
$$

where the new unknown function is

$$
U(\zeta, t)=\int_{0}^{\zeta} S(\eta, t) d \eta
$$

A loss of smoothness of the generalized solution ${ }^{23}$ to Equation (36) determines the possibility of shock waves. Normally, the relative permeability coefficient is a convex function of saturation. Using set-valued analysis methods, see e.g., Refs., ${ }^{24,25}$ one can demonstrate that shocks cannot develop if $U$ is a concave function of $\zeta$. The function $U(\zeta)$ is concave if water saturation increases with depth. In other words, shocks do not develop if the gas saturation is higher in the more elevated parts of the plume. In those parts of the plume, where water saturation increases with depth, developing shocks may break the plume into parts.

These general observations can be inspected more closely using method of characteristics. Clearly, the characteristics of Equation (35) are straight lines and the saturation is constant along the characteristics. Therefore, the higher water saturation is, the steeper is the slope of the respective characteristic. In the top part of the plume, where water saturation decreases with depth, the characteristics of Equation (35) diverge and shocks cannot develop. If the saturation is constant over some interval inside the plume, the respective characteristics are parallel to each other. In other words, a part of the plume resembling a contiguous column of constant saturation migrates upward almost like a rigid body. However, such flow is unsteady and very likely this part of the plume will break. The plume instability is illustrated in Fig. 2. In computations, the relative water permeability is taken in the form (A.5). At $\tau=0$, a small deviation from constant water saturation is modelled by a sinusoid with a small amplitude. The sinusoidal form of the initial perturbation has been selected for the sake of simplicity. With time, the sinusoid is first deformed $(\tau=1.5)$ and then a shock appears $(\tau=3.5)$. As the shock develops, assumption (34) becomes invalid and one must use the full equation (33) or its nonlinear parabolic approximation (see Equation (41)) employing a different time scaling.

Another extreme situation takes place if

$$
\left|\gamma \mathcal{J}^{\prime}(S) \frac{\partial S}{\partial \zeta}\right| \gg 1
$$



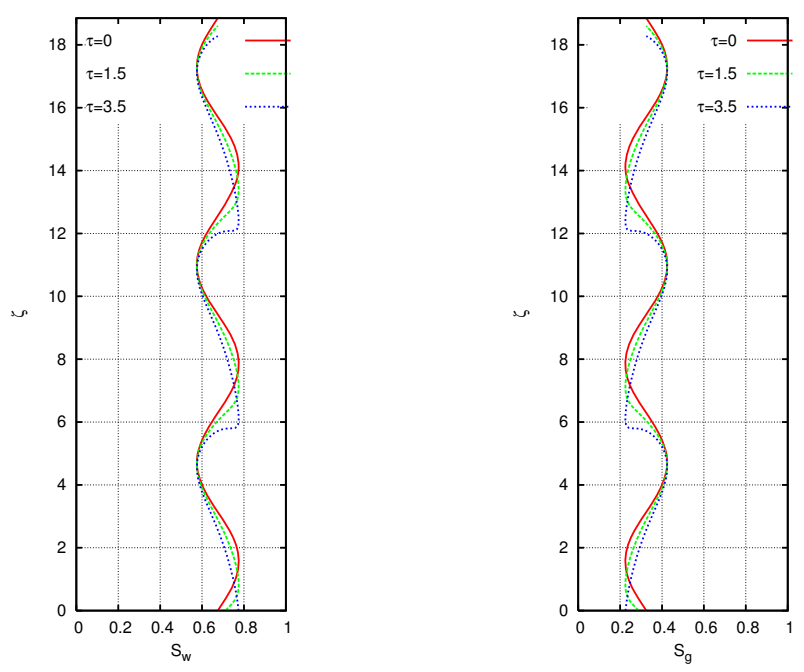

Fig. 2 - Development of shocks on vertical water (left) and gas (right) saturation profiles.

Such a condition is likely to be satisfied at the gas-water interface near the leading front of the plume, where in a relatively narrow zone the saturation variation is large. The specific size of such a zone depends on the character of the dependence of the capillary pressure on water saturation near the capillary entry pressure. In any case, such a zone would be larger inside a low-permeability seal than in permeable aquifer sand, due to the presence of permeability coefficient in the denominator of $\gamma$, Equation (32). Estimate (38) leads to Ryzhik's equations of countercurrent imbibition $^{18}$

$$
\phi \frac{\partial S}{\partial \tau}=-\frac{\partial}{\partial \zeta}\left(\gamma f(S) \mathcal{J}^{\prime}(S) \frac{\partial S}{\partial \zeta}\right)
$$

For the latter equation, it is convenient to modify the time scale by defining a new dimensionless time

$$
\vartheta=\frac{\gamma}{\phi} \tau=\frac{\sigma}{\mu_{w} H^{2}} \sqrt{\frac{k}{\phi}} t
$$

The scaling coefficient of $t$ on the right-hand side measures the ratio between capillary and viscous forces. In terms of dimensionless time $\vartheta$, Equation (39) takes on the form

$$
\frac{\partial S}{\partial \vartheta}=\frac{\partial^{2}}{\partial \zeta^{2}} \Phi(S)
$$

where

$$
\Phi(S)=-\int_{S_{*}}^{S} f(\eta) \mathcal{J}^{\prime}(\eta) d \eta
$$

Here $S_{*}$ is residual water saturation. Ryzhik, ${ }^{18}$ see also, ${ }^{21}$ has demonstrated that this equation admits a self-similar solution, which is discussed below. Note, that in this asymptotic case, gravity does not enter the differential equation, so the latter is governed by capillarity and viscous flow only.
In a low-permeability ( $k \ll 1$ Darcy) thin layer, the conversion coefficient between $\tau$ and $\vartheta$ is greater than one. In a highly-permeable thick aquifer (hundred meters), the opposite picture is true. Near the developing shocks, Fig. 2, the gradient of saturation increases significantly, making the capillary pressure the prevailing force. Consequently, the velocity of propagation at the developing shocks decays approximately as $\frac{1}{\sqrt{\vartheta}}$, and because of the difference of velocities in different parts, the plume is likely to break into parts. The size of each "sub-plume" is determined by the relative permeability and capillary pressure curves and the density contrast between gas and water. Again, due to the different velocities of propagation, which may be the case even in a homogeneous reservoir, plumes can coalesce with one another. Once such a conglomerate plume exceeds a certain critical vertical dimension, the reservoir seal can be penetrated through most permeable parts, e.g., a system of cracks. Such a penetration through a sequence of low-permeability seals with defects has been considered in some numerical simulations. ${ }^{26}$ In the next section, we analyze the propagation of a plume in a low-permeability seal.

\section{Countercurrent flow in a low-permeability seal}

In vertical migration resulting from a gas storage leak, the gas flows through alternating formation layers of high and low permeability, and of different thicknesses. The force driving this flow is buoyancy. The process is similar to secondary hydrocarbon migration and trapping. ${ }^{9}$ If a connected gas plume extends between depths $z_{\text {bottom }}$ and $z_{\text {top }}$, then, at hydrostatic conditions, the buoyancy force is equilibrated by the capillarity:

$$
\left(\varrho_{w}-\varrho_{g}\right)\left(z_{\text {bottom }}-z_{\text {top }}\right) g d z=p_{c}\left(z_{\text {top }}\right)-p_{c}\left(z_{\text {bottom }}\right)
$$

cf Equation (19). At the top, the capillary pressure is bounded by the capillary entry pressure. Inside the plume, and at the bottom, the capillary pressure is controlled by the water saturation in combination with hysteretic effects, see e.g., Ref. ${ }^{14}$ The same factors regulate the largest feasible magnitude of the difference on the right-hand side of Equation (43). The maximun value of this difference determines the maximal feasible vertical extent of the plume, $z_{\text {top }}-z_{\text {bottom }}$. Consider a situation where the top of the plume contacts a low-permeability formation layer, where the drainage entry pressure is high, Fig. 3. If continued gas leakage from a deeper storage aquifers increases the vertical size of the plume. As the buoyancy force becomes larger than the capillary-pressure constraint on the righthand side of Equation (43), the gas can penetrate into the seal, Fig. 3.

The seal rock is tight relative to the underlying layer. In the latter, the vertical extent of the connected plume is much larger than the thickness of the seal. In addition, the porosity in the seal is much smaller than that of the underlying formation. Therefore, the volume of gas that has already entered the seal is much smaller than the amount 


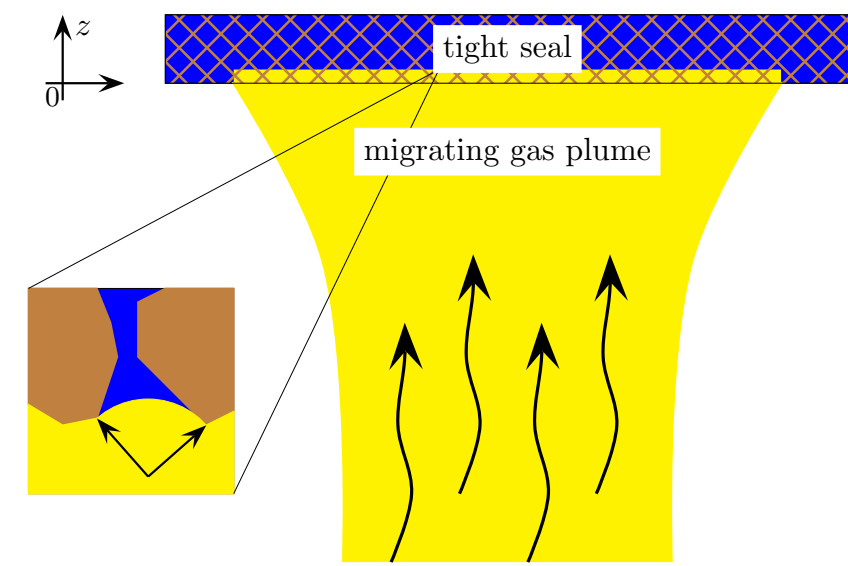

Fig. 3 - Gas propagation through a seal. The seal cannot be penetrated until the gas-liquid menisci become small enough by increasing capillary pressure.

of gas in the plume. Relative to the volume of the gas that has entered the seal, the rest of the plume can be considered as an infinite source of gas. Thus, for the following analysis, the gas pressure and water saturation at the seal bottom boundary, $p_{g s b}$ and $S_{g s b}$, are assumed constant.

Detailed resolution of the saturation profile at the very tip of the plume requires information about the behavior of the gas relative permeability and capillary pressure curves near full water saturation, $S=1$, see Ref. ${ }^{19}$ Such information cannot be easily obtained experimentally. To get around this difficulty, we define an entry saturation, $S_{e}<1$, the largest saturation of water, at which the relative permeability to gas is appreciably non-zero. We associate the dimensionless vertical coordinate of the top boundary of the gas plume, $\zeta_{t o p}(\vartheta)$, with the location near the tip of the plume where the saturation equals $S_{e}$. There is a certain arbitrariness in the definition of exact value of $S_{e}$. However, this arbitrariness affects the fluid saturation of a small region near the tip of the plume only. ditions:

Thus, one obtains the following pair of boundary con-

$$
S\left(\zeta_{s b}, \vartheta\right)=S_{g s b}, \quad S\left(\zeta_{t o p}(\vartheta), \vartheta\right)=S_{e}
$$

One more boundary condition at the top boundary of a plume migrating upward is implied by mass conservation. Above the plume, the liquid saturation is identically equal to unity and there is no gas flow through the moving top boundary of the plume. Between times $\vartheta$ and $\vartheta+\delta \vartheta$, where $\delta \vartheta$ is a small time increment, the boundary will move by the distance

$$
\begin{aligned}
\zeta_{\text {top }}(\vartheta+\delta \vartheta)-\zeta_{\text {top }}(\vartheta) & =\int_{\vartheta}^{\vartheta+\delta \vartheta} \frac{d}{d \vartheta} \zeta_{t o p}\left(\vartheta^{\prime}\right) d \vartheta^{\prime} \\
& \approx \frac{d}{d \vartheta} \zeta_{t o p}(\vartheta) \delta \vartheta
\end{aligned}
$$

The gas saturation integral between $\zeta_{\text {top }}(\vartheta)$ and $\zeta_{\text {top }}(\vartheta+$ $\delta \vartheta)$ is equal to

$$
\begin{aligned}
& \int_{\zeta_{t o p}(\vartheta)}^{\zeta_{t o p}(\vartheta+\delta \vartheta)}\left(1-S\left(\vartheta+\delta \vartheta, \zeta^{\prime}\right)\right) d \zeta^{\prime} \\
& \approx\left(1-S_{e}\right) \frac{d}{d \vartheta} \zeta_{t o p}(\vartheta) \delta \vartheta
\end{aligned}
$$

Equations (45) and (46) are accurate up to higher order terms with respect to $\delta \vartheta$.

Because of the countercurrent flow, gas saturation increase is equally compensated by the flow of the brine downwards through the boundary at $\zeta=\zeta_{\text {top }}(\vartheta)$, which, according to Equation (41) is measured by the integral

$$
\begin{array}{r}
-\int_{\vartheta}^{\vartheta+\delta \vartheta} \frac{\partial}{\partial \zeta} \Phi\left(S\left(\vartheta^{\prime}, \zeta_{\text {top }}(\vartheta)\right)\right) d \vartheta^{\prime} \\
\approx-\left.\frac{\partial}{\partial \zeta} \Phi^{\prime}(S)\right|_{S=S_{e}} \delta \vartheta
\end{array}
$$

Thus, equating (46) and (47) and passing to the limit as $\delta \vartheta \rightarrow 0$, one obtains:

$$
\left(1-S_{e}\right) \frac{d}{d \vartheta} \zeta_{t o p}(\vartheta)=-\left.f(S) \mathcal{J}^{\prime}(S)\right|_{S=S_{e}} \frac{\partial S}{\partial \zeta}
$$

The last equation expresses the mass balance at the top boundary of the plume in differential form.

Equation (41) admits a self-similar solution satisfying the boundary conditions (44) and (48). Indeed, define a similarity variable by

$$
\xi=\frac{\zeta-\zeta_{s b}}{\sqrt{\vartheta}}
$$

Then, for

$$
\zeta_{\text {top }}(\vartheta)=\zeta_{s b}+a \sqrt{\vartheta}
$$

one arrives at the following boundary-value problem for an ordinary differential equation for $S(\zeta, \vartheta)=S(\xi)$ :

$$
\begin{aligned}
-\frac{\xi}{2} \frac{d S}{d \xi} & =\frac{d^{2}}{d \xi^{2}} \Phi(S) \\
S(0) & =S_{g s b}, \quad S(a)=S_{e} \\
\frac{1}{2}\left(1-S_{e}\right) a & =\left.\left.\Phi^{\prime}(S)\right|_{S=S_{e}} \frac{d S}{d \xi}\right|_{\xi=a}
\end{aligned}
$$

The boundary-value problem (51)-(53) involves a secondorder differential equation with three boundary conditions and an unknown scalar parameter $a$. Such a problem often is called a nonlinear eigenvalue problem. It can be solved, for example, using a modification of the shooting method. ${ }^{27}$

Equation (51) is of second order. To solve it, we transform it into a system of first-order equations by introducing a new variable

$$
W=\frac{d}{d \xi} \Phi(S)
$$




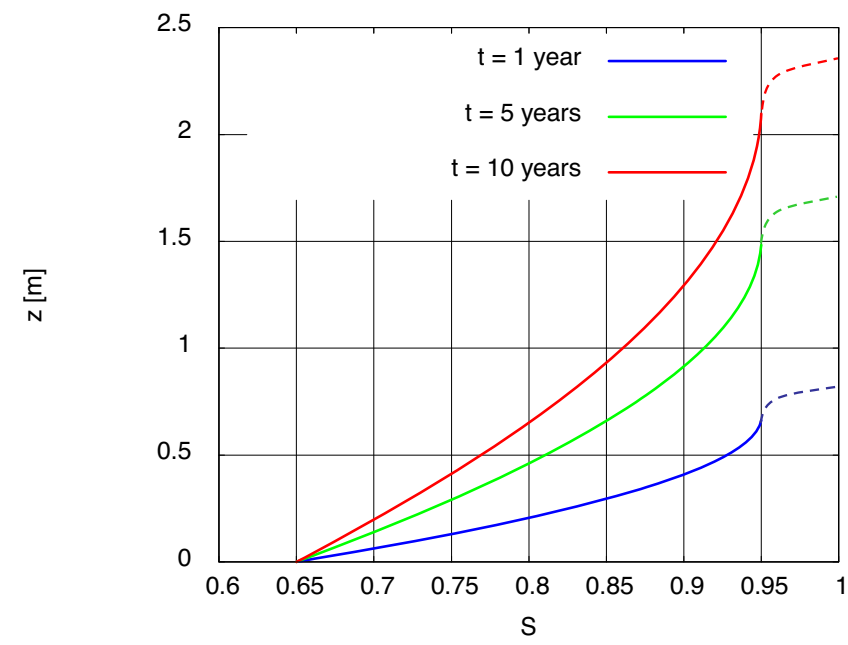

Fig. 4 - Ryzhik's self-similar solution: water saturation profiles for several different times. Water saturation at the boundary is assumed equal to $65 \%$. Saturation profile near the very tip of the plume is not resolved and is presented approximately with a dashed line.

Thus, we need to solve the system

$$
\left\{\begin{aligned}
\frac{d}{d \xi} S & =\frac{1}{\Phi^{\prime}(S)} W \\
\frac{d}{d \xi} W & =-\frac{\xi}{2 \Phi^{\prime}(S)} W
\end{aligned}\right.
$$

with boundary conditions

$$
S(0)=S_{g s b}, \quad S(a)=S_{e}, \quad \text { and } \quad W(a)=\frac{1}{2}\left(1-S_{e}\right) a
$$

The similarity saturation distribution has been computed based on formulae (A.1), with $\lambda_{P_{c}}=0.3$, and (A.5), with $\lambda_{k_{r w}}=0.457$. The water saturation at the boundary of the seal is supposed to be $65 \%$.

In the original dimensional variables, $z$ and $t$, the saturation profiles can be easily obtained from Ryzhik's solution. The result is shown in Fig. 4. The relative permeability and capillary pressure functions, as well as the other parameters used in this calculation are presented in Appendix at the end of this paper.

\section{Countercurrent flow in a permeable aquifer}

Under buoyancy, a plume of gas, created by a leaking gas storage reservoir, migrates upwards. In this section, we analyze a model of such migration. It turns out that there are at least two stable zones, at the top and at the bottom of the plume. The migration of each of these parts is characterized by a travelling-wave solution. In other words, the water and gas saturation distributions inside these stable zones do not change as they migrate upwards.

Local heterogeneities of the formation impact the structure of the stable zones. A leak from gas storage

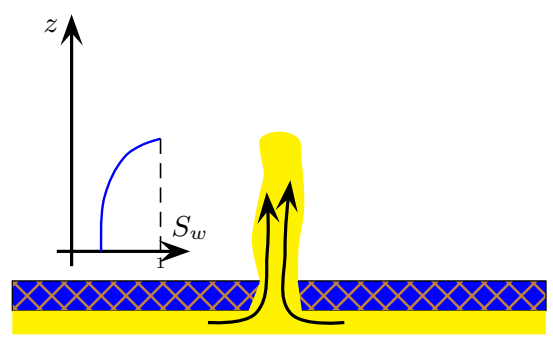

Fig. 5 - Leak of gas through a seal: continuous stream

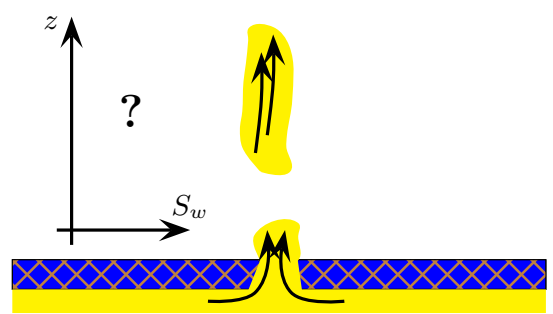

Fig. 6 - Leak of gas through a seal: flow in separate plumes

can be intense enough to support a continuous streamlike flow, Fig. 5. However, if the gas plume enters a high-permeability zone, the top of the plume may run away and break a plume into separate parts, Fig. 6. In this study, we focus on characterizing the structure of the above-mentioned stable zones of individual plumes.

We seek a travelling-wave solution to Equation (33). Let $v$ be the velocity of propagation, so that we seek a solution, which can be presented as a function of a single composite variable $z-t v$. From Equation (24),

$$
v \phi S^{\prime}=\frac{k}{\mu_{w}}\left(f(S)\left(\frac{d p_{c}(S)}{d S} S^{\prime}-\left(\varrho_{w}-\varrho_{g}\right) g\right)\right)^{\prime}
$$

Here the prime denotes the derivative with respect to the composite variable. Define dimensionless plume propagation velocity $V$ as

$$
V=\frac{\mu_{w}}{k\left(\varrho_{w}-\varrho_{g}\right) g} v
$$

Then, in dimensionless variables, we seek a solution in the form

$$
S(\zeta, \tau)=\Psi(\xi)
$$

where

$$
\xi=\zeta-\tau V
$$

A dimensionless travelling wave solution satisfies the equation

$$
\phi V \frac{d \Psi}{d \xi}-\frac{d}{d \xi}\left(f(\Psi)\left(\gamma \mathcal{J}^{\prime}(\Psi) \frac{d \Psi}{d \xi}-1\right)\right)=0
$$


The plume migration velocity, $v$, can be found from the following considerations. Let us associate $\xi=0$ with the location in the plume corresponding to the minimum water saturation. The argument in the previous section suggests that due to the local heterogeneities there might me several local minima of saturation alternating with local maxima, Fig. 2. Let us focus on the top and the bottom ones. The structure of the plume between these two water saturation minima can be complex, so that a realistic detailed picture may require high-accuracy numerical simulations. fore,

At $\xi=0$, water saturation attains its minimum, there-

$$
\Psi^{\prime}(0)=0
$$

Thus, for dimensionless water Darcy velocity, Equation (31) implies

$$
W_{v}=-f(\Psi(0))
$$

The top part of the plume, between the minimum water saturation and the tip of the plume, flows upward with velocity $v$. Therefore, the volume of gas crossing a unit area in a horizontal cross-section over a small time interval $\triangle \tau$ is approximately equal to $v(1-\Psi) \phi \delta \tau$. This incremental amount of gas is the result of Darcy flow. Therefore, it is equal to $-u_{w} \delta \tau$. Thus, in dimensional velocities, on obtains

$$
v(1-\Psi(0)) \phi=-u_{w}
$$

For the dimensionless velocity, using Equations (63), one obtains

$$
V(S)=\left.\frac{f(S)}{\phi(1-S)}\right|_{S=\Psi(0)}
$$

Equation (65) defines the velocity of plume migration as a function of minimal brine saturation. The plot of this function is shown in Fig. 7. The function $V(S)$ has a maximum, which defines the theoretical upper limit for the velocity of plume migration. The sharp shape of the plot is the consequence of the significant viscosity contrast between water and gas. In case of hydrocarbon migration, this contrast may be significantly lower. Therefore, the respective curve will be smoother and the maximum will shift towards a lower water saturation. Fig. 8 shows three plots of $v(S)$ evaluated in physical units for different viscosity ratios. In all three plots all parameters are the same except for the viscosity of the less dense non-wetting fluid. In particular, the absolute permeability is 100 millidarcy and the porosity is $20 \%$.

Plume stability requirement imposes certain constraints on the diustribution of saturation inside the plume. These requirements can be formulated by analyzing the shape of the curve $V(S)$. Clearly, if different parts of the plume propagate with different velocities, the saturation distribution inside the plume cannot be constant. For example, if the upper part of the plume moves faster than the lower part, such a plume will eventually break into parts, Fig. 6. In contrast, if the lower part of the plume moves faster, then the resulting accumulation of the gas inside

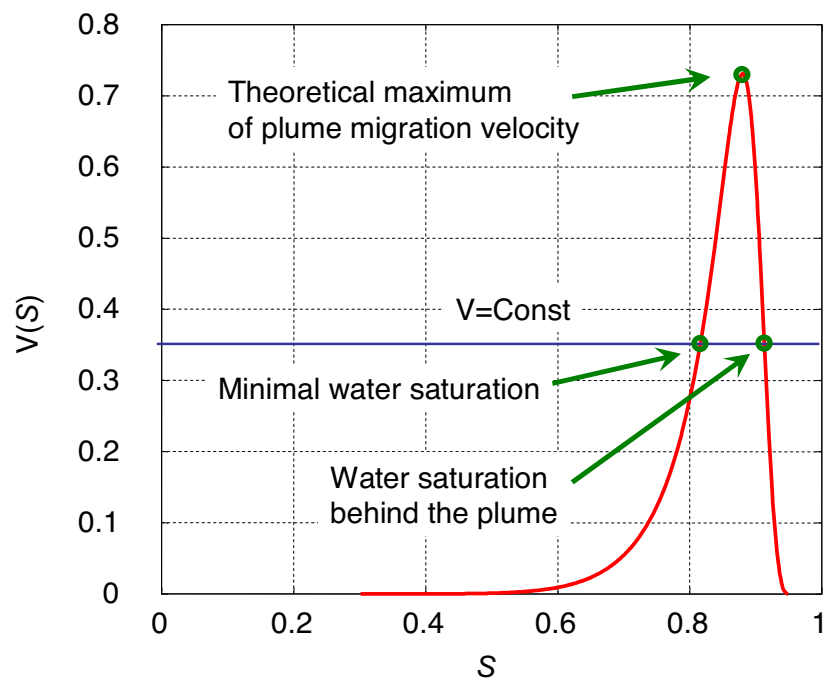

Fig. 7 - The top of the $V(S)$ curve defines the theoretical maximum of the plume propagation velocity.

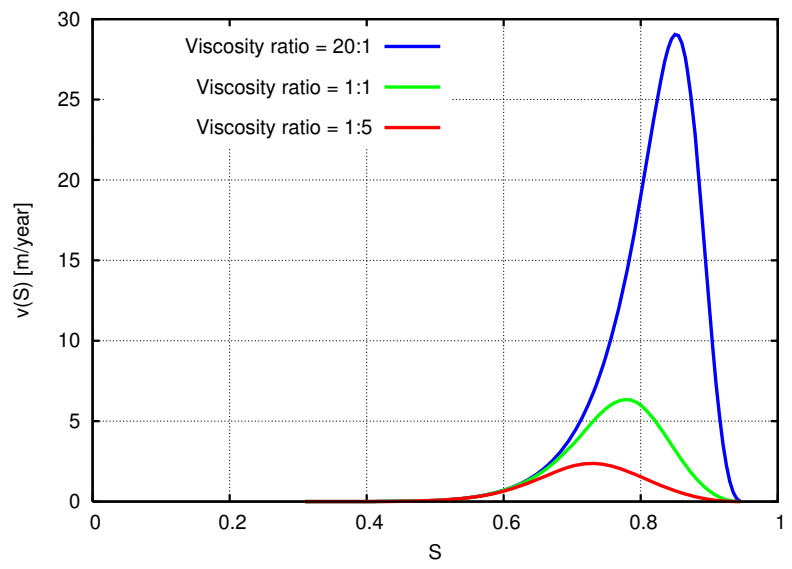

Fig. 8 - Plume migration velocity in $\mathrm{m} /$ year for three different viscosity ratios.

the plume will result in a saturation discontinuity, like a shock wave as shown in Fig. 2. Such a periodic plume with shocks can be viable only if the velocity (65) evaluated at local maxima and minima of saturation are equal. In Fig. 7, such a pair of saturations must correspond to two intersections of the plot with a horizontal straight line. In particular, this means that such a periodic plume propagates with a velocity, which is smaller than the maximum value of $V(S)$.

Analysis of the dependence $V(S)$ suggests possibility of two stable zones: at the top and at the tail of a plume. Between these zones, simulation of the transient saturation redistribution may require numerical simulations. In the next subsections we focus on these two stable zones. 
Propagation of the top of the plume. Consider first only the upper part of the plume, where water saturation monotonically decreases with depth. At the very tip of the plume, the capillary pressure is equal to the entry capillary pressure. As in Equation (44), denote by $S_{e}$ the respective water saturation and let $v$ be the velocity of propagation at the top part of the plume. The volumetric flux of water and gas near the tip of the plume can be expressed through Darcy velocity and the speed of plume propagation. Hence, by virtue of Equation (16), one obtains

$$
v\left(1-S_{e}\right) \phi=\frac{k}{\mu_{w}} f\left(S_{e}\right)\left[-\frac{\partial}{\partial z} P_{c}(\Psi)+\left(\varrho_{w}-\varrho_{g}\right) g\right]
$$

where the right-hand side of this equation is evaluated at the tip of the plume. In dimensionless form,

$$
\phi V(1-\Psi)=-f(\Psi)\left(\gamma \mathcal{J}^{\prime}(\Psi) \frac{d}{d \xi} \Psi-1\right)
$$

The last equation can be also obtained by integration of Equation (61).

The dimensionless velocity $V$ on the left-hand side of Equation (67) is the velocity of propagation of the top part of the plume. It can be denoted by $V_{\text {top }}$ to distinguish from $V(S)$ formally calculated from Equation (65). Using the latter, one obtains

$$
\gamma \mathcal{J}^{\prime}(\Psi) \frac{d \Psi}{d \xi}=1-\frac{V_{\text {top }}}{V(\Psi)}
$$

The derivative of Leverett's function is negative. The water saturation is at maximum near the tip of the plume and decreases downward. Therefore, the left-hand side of the last equation is nonpositive. Consequently, $V(S)$ evaluated anywhere in this part of plume is lesser or equal to the velocity of propagation $V_{\text {top. }}$. Note that in this case, $V(S)$ is a dimensionless velocity evaluated using formula (65) and is not related to the physical velocity of the plume propagation.

The relationship

$$
\left.\frac{d \Psi}{d \xi}\right|_{\text {top }}=\frac{1-\frac{V_{\text {top }}}{V\left(S_{e}\right)}}{\gamma \mathcal{J}^{\prime}\left(S_{e}\right)}
$$

characterizes the slope of the saturation profile at the tip of the plume. According to Fig. 7, the numerator of the fraction on the right-hand side grows rapidly as $S_{e}$ approaches the end-point water saturation. In other words, the water saturation drops abruptly at the leading edge of the plume. Fig. 9 shows the water saturation profile in the top part of the plume at different times. The plots have common vertical asymptotes. Indeed, as the value of $V(\Psi)$ approaches $V_{\text {top }}$, the right-hand side of equation (68) vanishes and the saturation becomes almost constant with respect to depth. We must assume that the saturation $S_{e}$ corresponding to the capillary entry pressure is to the right from the saturation corresponding to the theoretical maximum of the plume propagation velocity, Fig. 7.

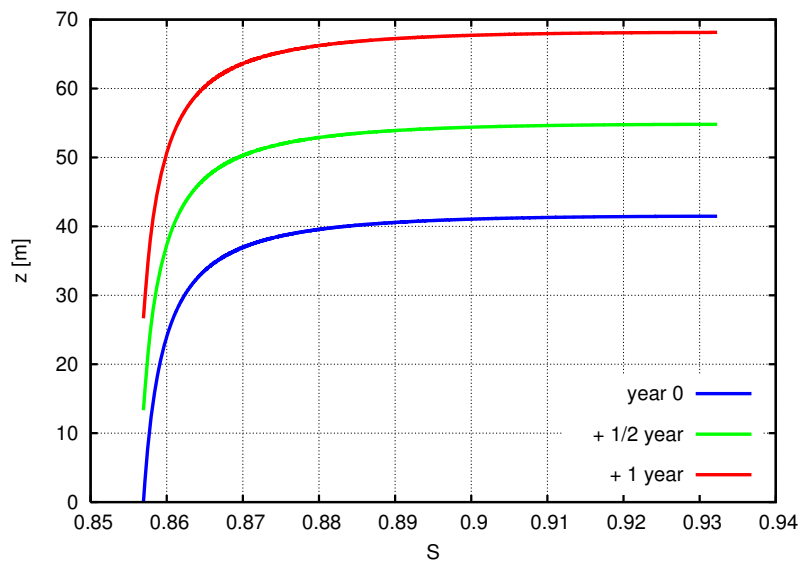

Fig. 9 - Distribution of the saturation near the top of the plume

The Cauchy problem for Equation (68) can be solved with the end-point condition $\Psi=S_{e}$ at the top. It is convenient to flip the independent and unknown variables and to write this equation down in the form

$$
\frac{d \xi}{d \Psi}=\frac{\gamma \mathcal{J}^{\prime}(\Psi)}{1-\frac{V_{\mathrm{top}}}{V(\Psi)}}
$$

The latter can be solved by integration

$$
\xi=\xi_{\text {top }}-\int_{\Psi}^{S_{e}} \frac{\gamma \mathcal{J}^{\prime}(S)}{1-\frac{V_{\text {top }}}{V(S)}} d S
$$

Fig. 9 shows an example of calculations. Once the saturation profile is available, the gradient of water saturation can be evaluated re-using Equation (68). Then, Darcy velocity is readily obtained by substitution of the results into Equation (31). Finally, the water pressure is obtainable by converting the dimensionless Darcy velocity back into physical units and integrating Equation (15). The gas pressure is evaluated using capillary pressure, see Equation (1). Figs. 10 and $\mathbf{1 1}$ show results of such calculations. Note that the flows are very slow and the water pressure profile only slightly deviate from the hydrostatic one. The capillary pressure is constant at constant water saturation, so in the part of the plume where the saturation does not significantly vary the gas pressure gradient is close to that of water.

The remaining unresolved parameter is $V_{\text {top }}$. Substitution of any value between $V\left(S_{e}\right)$ and the maximal theoretical velocity in the above-presented calculations produces a mathematically feasible solution. It seems, however, that not any one of such solutions is viable for a long period of time. Note, that the water saturation in the top part of the plume is higher upwards. On the opposite, the respective value of velocity $V(S)$ increases from the very 


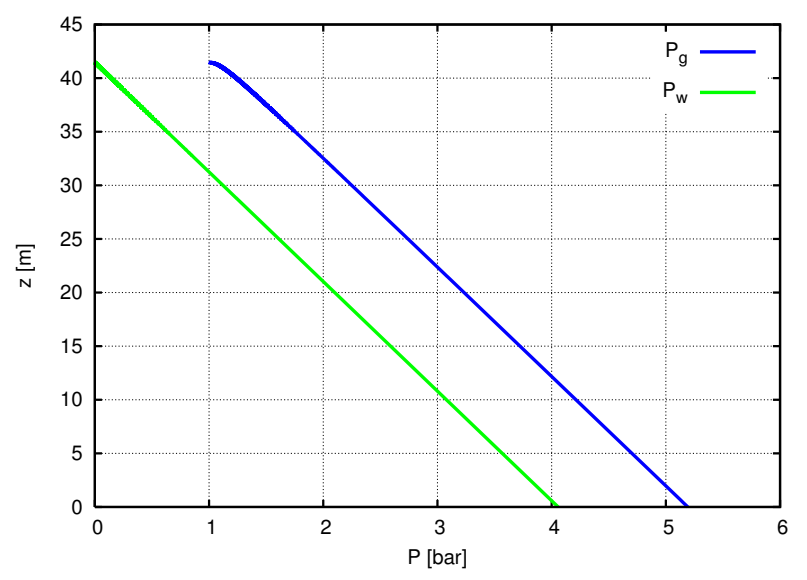

Fig. 10 - Except the very top of the plume, where large variation of saturation creates significant gradient of capillary pressure, the pressure profile almost is almost hydrostatic. Pressures are evaluated relative to the water pressure at the top of the plume.

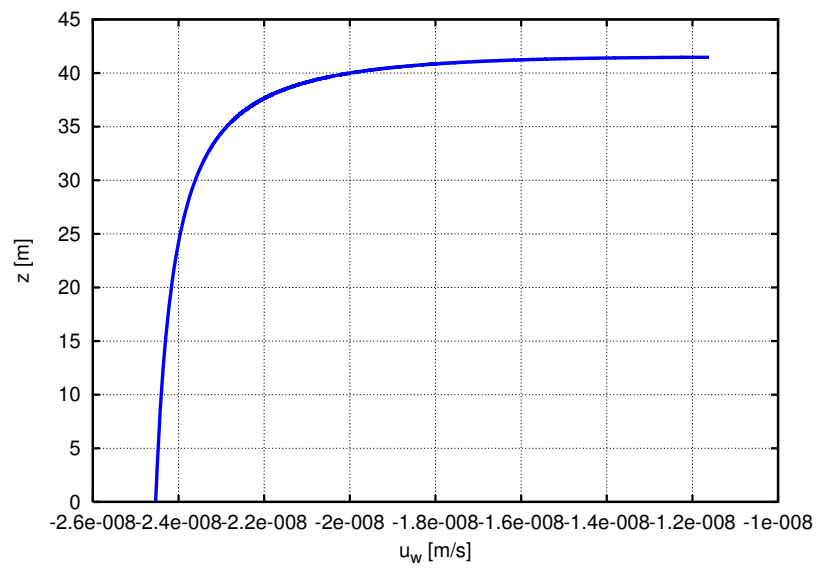

Fig. 11 - The profile of Darcy velocity of water at the top of the plume almost mimics that of saturation. top downwards. Local heterogeneities may create saturation fluctuations. Such fluctuations, in turn, may create parts of the plume with a reduced, relative to the ideal profile, saturation. If the resulting velocity, $V(S)$, exceeds the velocity of propagation, $V_{\mathrm{top}}$, then a part of plume can break out and leave the remaining part behind, Fig. 6 . The likelihood of such a scenario apparently is greater over a longer period of time. However, such separation of the plume is prohibited, if $V_{\text {top }}$ is equal to the theoretical maximum, Fig. 7. In this case, no part of the plume can run away from the rest. Moreover, the parts migrating with the maximal theoretical velocity push the slower ones, and maintain the integrity of the top part of the plume.

Saturation distribution in the tail of the plume. At the plume tail, water saturation is a decreasing function of $\xi$. With depth, its value approaches the maximal saturation, at which gas cannot flow. Let us associate $\xi=0$ with the deepest location in the plume where water saturation attains its minimum.

The fluid displacement at the bottom part of the plume is imbibition, where the wetting fluid displaces the nonwetting one. The relative permeability and capillary pressure functions in imbibition are different from those in drainage. In addition, non-equilibrium effects can affect the dynamic of imbibition as well. ${ }^{17,19}$ In this study, we assume that the the time of redistribution of the fluids is small and nonequilibrium effects can be neglected. In our case, this assumption is justified by the slowness of the buoyancy-driven migration and the relatively low viscosity of the nonwetting fluid. Since we use generic relative permeability and capillary pressure curves anyway, see Appendix, we do not account for hysteresis effect as well.

Similarly to Equation (70), flipping $\Psi$ and $\xi$ in Equation (67), one obtains

$$
\frac{d \xi}{d \Psi}=\frac{\gamma \mathcal{J}^{\prime}(\Psi)}{1-\frac{V(\Psi(0))}{V(\Psi)}}
$$

The sign of the derivative $\frac{d \Psi}{d \xi}$ is the same as the sign of $\frac{d \xi}{d \Psi}$. The derivative of the Leverett's function is always negative. Hence, the saturation increases with $\xi$ if $V(\Psi(0))>V(\Psi)$, and decreases with $\xi$ if $V(\Psi(0))<V(\Psi)$. According to our assumptions, $\Psi(0)$ is the minimal water saturation in the plume and, therefore, inequality $V(\Psi(0))>V(\Psi)$ must hold true. In particular, $\Psi(0)$ must be smaller than the value of saturation corresponding to the maximum of $V(S)$. In such a case, the saturation is a decreasing function of $\xi$, which corresponds to the saturation distribution in the tail of the plume. The plot of the saturation distribution in the tail of the plume can be calculated by integration of Equation (72) in $\Psi$ :

$$
\xi(\Psi)=\gamma \int_{(1+\varepsilon) \Psi(0)}^{\Psi} \frac{\mathcal{J}^{\prime}(S)}{1-\frac{V(\Psi(0))}{V(\Psi)}} d S
$$




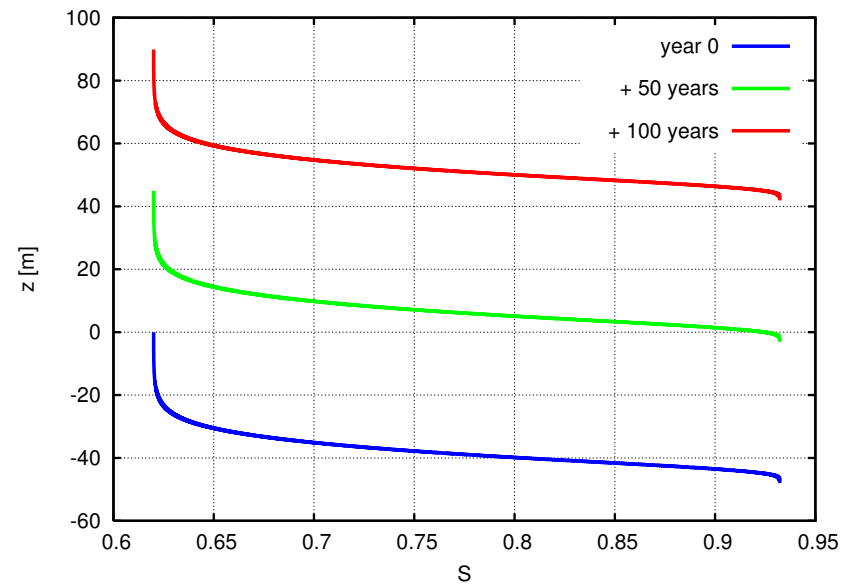

Fig. 12 - Distribution of water saturation in the tail of the plume. Note the difference in the time scale relative to the top of the plume, Fig. 9.

The denominator of the expression inside the integral on the right-hand side vanishes at $\Psi \rightarrow \Psi(0)$. This means that the value of $\Psi(0)$ is not attainable on a finite interval. However, in reality, there are always some fluctuations of the saturation. In addition, the travelling-wave solution is an asymptotic idealization. Therefore, we apply Equation (72) in an intermediate region, where the saturation is less than the saturation behind the plume, but larger than $\Psi(0)$, the minimal saturation inside the plume. A tolerance factor, $\varepsilon$, has been introduced to avoid division by zero. Fig. 12 shows an example of such calculation.

These calculations along with the plot of the plume migration velocity lead to certain conclusions. If the minimal brine saturation in the plume corresponds to a point on the rising part of the curve $V(S)$ in Fig. 7, then the saturation at the tail of the plume is determined by the second intersection of the horizontal line corresponding to the plume propagation velocity. The low gas saturation zone behind the plume cannot be included in the stable travelling-wave part. This conclusion is implied by comparison of the signs of the expressions one both sides of Equation (72).

As in the previous section, once the saturation profile has been calculated, the Darcy velocity, and gas and water pressures can be obtained from equations (31) and (15). Figs. 13 and 14 show results of such calculations. Again, since the flows are very slow, the pressure profiles for both gas and water only slightly deviate from the hydrostatic ones.

The closer the velocity of propagation of the plume to the theoretical maximum, the lesser is the difference between the minimal water saturation and the saturation behind the plume. Therefore, the tail of the plume propagates faster as both saturations approach the value corresponding to the maximum of $V(S)$. If the minimal water saturation is on the descending part of the curve in Fig. 7 ,

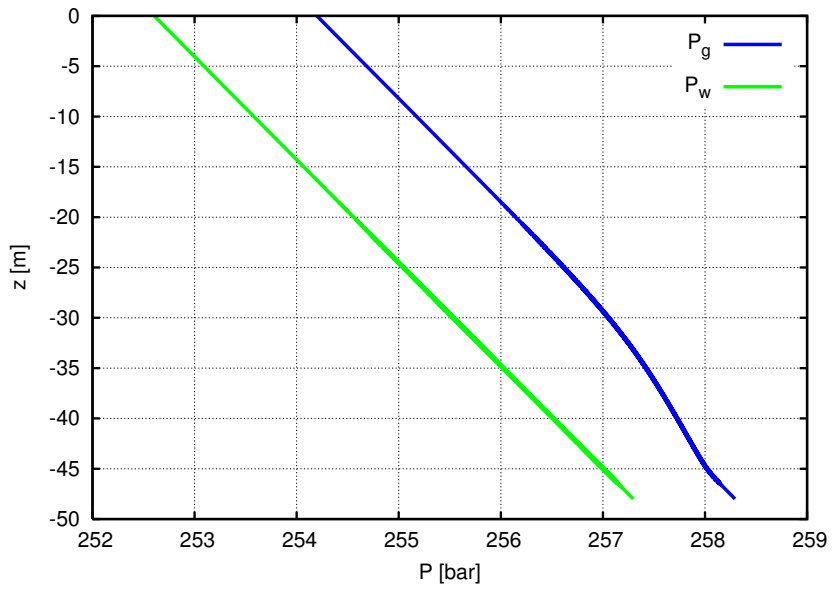

Fig. 13 - Gas and water pressures in the tail of a plume.

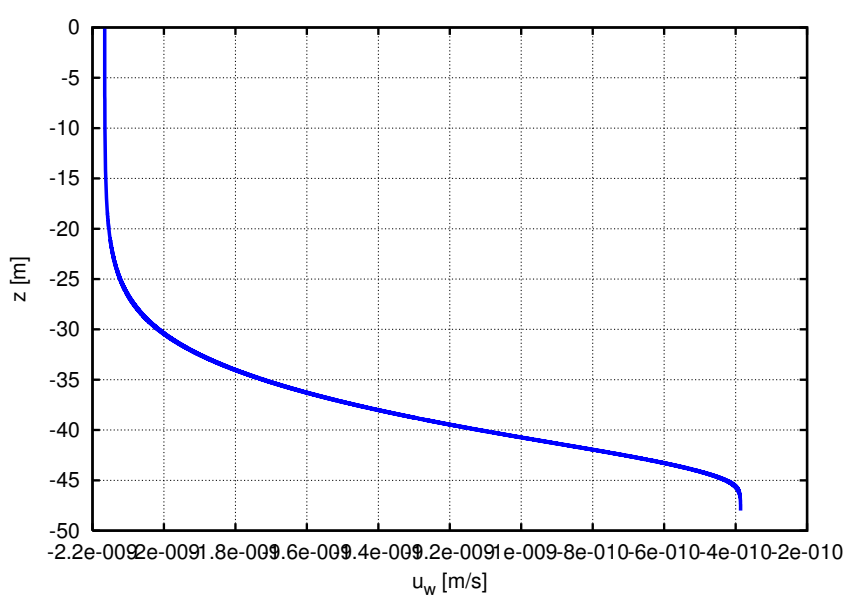

Fig. 14 - Darcy velocity in the tail of the plume. 
then such a plume most probably will break into a number of smaller plumes. Indeed, small fluctuations may create portions of the plume, where the variation of the saturation is small, so that the mass balance will lead to a local velocity of propagation, which is smaller than the velocity of the part of the plume above. As the leading part moves faster than the trailing one, the plume will likely break apart.

To conclude this subsection, we remark that the travelling-wave character of the bottom part of a gas plume can been observed in the numerical simulations reported in the book. ${ }^{8}$

\section{Conclusions}

The flow of gas leaking from a deep underground storage can take a form of migration of separate plumes. In this study, this flow is expressed as the result of the interactions of buoyant, capillary, and viscous forces. We consider only vertical flow, assuming either flow in a vertical fracture or flow in the middle part of a large plume. The two-phase flow is countercurrent, where an amount of gas flowing upward is replaced with an equal amount of water moving downward. The consequences of non-equilibrium and hysteresis effects will be studied elsewhere.

In a low-permeability medium, the gravity forces can be neglected and a self-similar solution similar to that obtained by Ryzhik ${ }^{18}$ can be applied. As the result, propagation of the leading portion of gas plume is scaled as square root of time.

In a general case of plume propagation in an aquifer, two stable zones of a gas plume have been identified: the top and the tail. In either one, the flow can be described as a travelling-wave propagation of the fluid saturation profile. The theoretical maximum of velocity of plume propagation can be determined from the dependence of this velocity on the minimal water saturation in the plume. This calculation leads to a simple rough estimate of time needed for the plume to reach the surface. The input parameters needed for this estimate are the vertical permeability log and the relative permeability curves. Simple calculations presented in this study suggest that the velocity of propagation of a plume of supercritical gas may reach values of the order of tens of meters per year in an aquifer whose permeability is of the order of 100 millidarcy.

The plume can be accelerated by local heterogeneities and slowed down by the dissolution of gas into water and large gas saturation left behind the plume. The velocity also accelerates closer to the surface due to the enhanced density and viscosity contrasts. Accurate prediction of the plume propagation velocity requires knowledge of the relative permeability and capillary pressure curves.

Analysis of two stable zones at the top and at the bottom of the plume suggests that the lower one has much higher gas saturation, but propagates with a much slower velocity, Fig. 1. Gas saturation in the top part of the plume is relatively small, which averages about $15 \%$ in the calculations presented in this study, but the velocity may be higher than that at the bottom of the plume by orders

\begin{tabular}{|l|c|}
\hline Absolute permeability in aquifer, $k$ & 100 millidarcy \\
\hline Absolute permeability in seal, $k$ & 0.01 millidarcy \\
\hline Porosity in aquifer, $\phi$ & $20 \%$ \\
\hline Porosity in seal, $\phi$ & $1 \%$ \\
\hline Water viscosity, $\mu_{w}$ & $1 . e^{-3} \mathrm{Pa-s}$ \\
\hline Gas viscosity, $\mu_{g}$ & $4.38 e^{-5} \mathrm{Pa-s}$ \\
\hline Water density, $\varrho_{w}$ & $1 . e^{+3} \mathrm{~kg} / \mathrm{m}^{3}$ \\
\hline Gas density, $\varrho_{g}$ & $0.561 e^{+3} \mathrm{~kg} / \mathrm{m}^{3}$ \\
\hline Surface tension coefficient, $\sigma$ & $70.0 e^{-3} \mathrm{~N} / \mathrm{m}$ \\
\hline
\end{tabular}

Table 1 - Formation and fluid parameters used in calculations.

of magnitude. The bottom part of the plume feeds the top part, as the latter rushes upward, like smoke from an open fire. The chances that the fast part of the plume will reach the surface before breaking apart due to insufficient gas supply from the bottom depend on the size of the leak and transport properties of the formation. Further studies will produce a more accurate picture of the processes involved and will lead to more accurate estimates of the time of migration and the likelihood of surface breakthrough.

\section{Acknowledgments}

This work was supported by the U.S. Department of Energy's Assistant Secretary for Coal through the Zero Emission Research and Technology Program under US Department of Energy contract no. DE-AC02-05CH11231 to Lawrence Berkeley National Laboratory.

The authors thank Dr. Christine Doughty of Lawrence Berkeley National Laboratory for reviewing the manuscript and providing very valuable remarks.

\section{Appendix}

Here we collect the formulae used for relative permeability and capillary pressure in the calculations.

The parameters characterizing the fluid and rock properties are collected in Table 1 . The gas properties roughly correspond to the properties of supercritical $\mathrm{CO}_{2}$ at the temperature of $60^{\circ} \mathrm{C}$ and the pressure of 150 bars.

For the Leverett's function, Equation (29), a modification of van Genuchten's formula ${ }^{28}$ has been used:

$$
\mathcal{J}(S)=\left(1-S^{* \frac{5}{\lambda}}\right)^{\lambda} S^{*-2 \lambda}
$$

where

$$
S^{*}=\frac{S-S_{i w}}{1-S_{i w}}
$$

The plot of such a curve for $S_{i w}=0.3, \lambda=0.3$ is presented in Fig. 15. Relative to a capillary pressure curve generated with van Genuchten's formula, the curve defined by Equation (A.1) corresponds to a relatively higher capillary-entry pressure in drainage.

The behavior of the capillary pressure and relative permeability curves near the endpoint saturations, where a two-phase flow gradually transforms into a single phase 


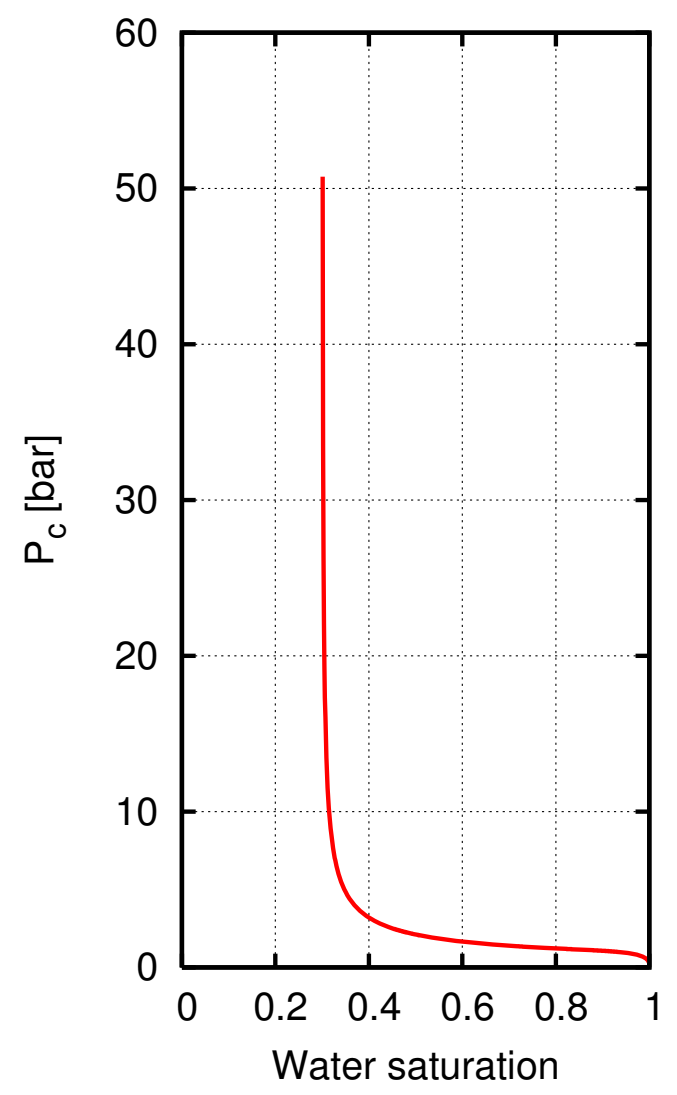

Fig. 15 - Capillary pressure curve: Leverett's $J$ function, Equation (29), is scaled using aquifer parameters.

flow, is subject to a great degree of uncertainty. A better understanding of this behavior will significantly benefit the predictive capabilities of the simulations. The curve in Fig. 15 qualitatively represents the point of view that the derivative of the capillary pressure curve goes to minus infinity as the gas saturation approaches $S_{i g}$.

For the gas relative permeability, a Corey type formula ${ }^{29}$ has been used:

$$
k_{r g}(S)=(1-\widetilde{S})^{2}\left(1-\widetilde{S}^{2}\right)
$$

where

$$
\widetilde{S}=\frac{S-S_{i w}}{1-S_{i g}-S_{i w}}
$$

In the calculations, the value of $S_{i g}=0.05$ has been used. With this choice of parameters, the product $\mathcal{J}^{\prime}(S) k_{r g}(S)$ tends to $-\infty$ as $S \rightarrow 1-S_{i g}$. If the latter condition is not satisfied, the solution becomes non-physical.

For computations, a van Genuchten water relative permeability function has been used

$$
k_{r w}(S)=\sqrt{S^{*}}\left[1-\left(1-S^{* 1 / \lambda}\right)^{\lambda}\right]^{2}
$$

where $S^{*}=S^{*}(S)$ is defined in Equation (A.2). The relative permeability to water drops at a relatively high satura-

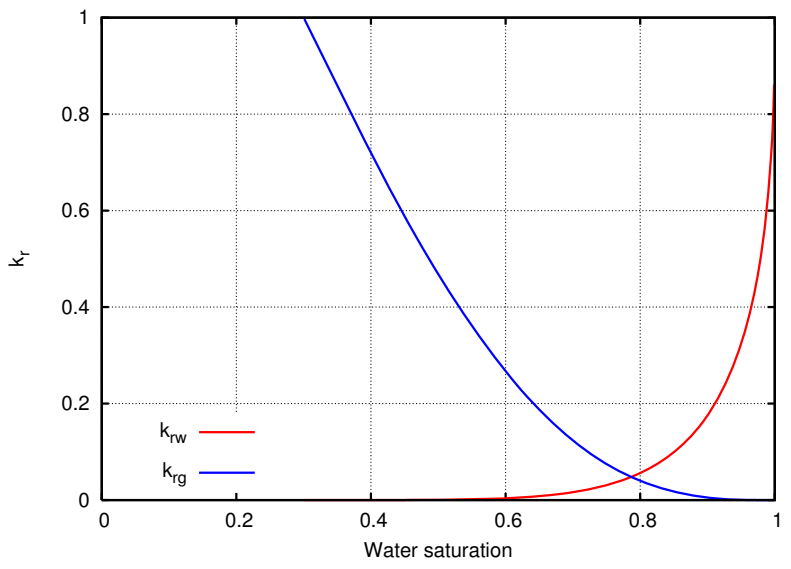

Fig. 16 - Gas relative permeability, (A.3) and water relative permeability (A.5) for $\lambda=0.457$, curves

tion, Fig. 16. This reflects the fact that water is the wetting fluid in our model and, therefore, it flows in the corners and the roughness of the pore walls. In other words, the wetting fluid has to flow in the part of the pore space, which provides the strongest resistivity to the flow.

\section{Nomenclature}

\section{Roman letters}

$f \quad$ fractional flow function

$g \quad$ gravity acceleration

$k \quad$ absolute permeability

$k_{r g} \quad$ gas relative permeability

$k_{r w} \quad$ water relative permeability

$\mathcal{J} \quad$ Leverett's J-function

$p_{c} \quad$ capillary pressure

$p_{g} \quad$ gas pressure

$p_{w} \quad$ water pressure

$S \quad$ water saturation

$S_{e} \quad$ drainage entry saturation

$S_{s b} \quad$ water saturation at the seal bottom boundary

$t \quad$ time

$u_{g} \quad$ gas Darcy velocity

$u_{w} \quad$ water Darcy velocity

$v \quad$ dimensional plume migration velocity

$V \quad$ dimensionless plume migration velocity

$W_{w} \quad$ dimensionless water Darcy velocity

$z \quad$ dimensional vertical coordinate

\section{Greek letters}

$\gamma \quad$ dimensionless factor

$\varrho_{g} \quad$ gas density

$\varrho_{w} \quad$ water density

$\zeta, \xi$ dimensionless vertical coordinate

$\sigma \quad$ surface tension coefficient

$\tau, \theta \quad$ dimensionless time

$\mu_{g} \quad$ gas viascosity

$\mu_{w} \quad$ water viscosity 
$\phi$

formation porosity

\section{References}

[1] IPCC, 2005. Intergovernmental Panel on Climate Change Special Report on Carbon Dioxide Capture and Storage. Cambridge University Press, Camdridge, United Kingdom, 2005.

[2] S. E. Gasda, S. Bachu, and M. A. Celia. The potential for $\mathrm{CO}_{2}$ leakage from storage sites in geological media: analysis of well distribution in mature sedimentary basins. Environmental Geology, 46(6-7):707-720, 2004.

[3] Jan Martin Nordbotten, Michael A. Celia, and Stefan Bachu. Injection and storage of $\mathrm{CO}_{2}$ in deep saline aquifers: Analytical solution for $\mathrm{CO}_{2}$ plume evolution during injection. Transport in Porous Media, 58(3):339-360, March 2005.

[4] E Lindeberg. Escape of $\mathrm{CO}_{2}$ from aquifers. Energy Conversion and Management, 38:235-240, 1997.

[5] C. Doughty and L. R. Myer. Scoping calculations on leakage of $\mathrm{CO}_{2}$ in geologic storage. In Brian McPherson and Eric Sundquist, editors, Science and technology of carbon sequestration, Washington DC, 2006. American Geophysical Union, in press.

[6] M. King Hubbert. Entrapment of petroleum under hydrodynamic conditions. The Bulletin of the American Association of Petroleum Geologists, 37(8):188-200, August 1953.

[7] M. Muskat. Physical Principles of Oil Production. McGraw-Hill, New York, NY, 1949.

[8] M. I. Shvidler and B. I. Levi. One-dimensional filtration of immiscible fluids (in Russian). Nedra, Moscow, 1970.

[9] F. I. Siddique and L. W. Lake. A comprehensive dynamic theory of hydrocarbon migration and trapping. SPE 38682. In 1997 72 $^{\text {th }}$ Annual Technical Conference and Exhibition, San Antonio, TX, 1997. SPE.

[10] P. Bedrikovetsky, J. De Deus, and J. Eurico Altoé. Secondary migration of oil: Analytical model. SPE 69411. In 2001 SPE Latin American and Carribean Petroleum Engineering Conference, Buenos Aires, Argentina, 2001. SPE.

[11] C. Doughty. Modeling geologic storage of carbon dioxide: comparison of non-hysteretic and hysteretic characteristic curves. In TOUGH Symposium, May 15-17 2006, Berkeley, CA, May 2006. Lawrence Berkeley National Laboratory.

[12] L. A. Rapoport. Scaling laws for use in design and operation of water-oil flow models. Trans. AIME, 204:143-150, 1955 .

[13] C. C. Mattax and J. R. Kyte. Imbibition oil recovery from fractured, water-drive reservoir. SPE Journal, 2:177-184, June 1962 .

[14] A. Al-Futaisi and T. W. Patzek. Impact of wettability on two-phase flow characteristics of sedimentary rock: Quasistatic model. Water Resources Research, 39(2):1042-1055, 2003.

[15] A. Al-Futaisi and T. W. Patzek. Secondary imbibition in NAPL-invaded mixed-wet sediments. J. Contaminant Hydrology, 74(1-4):61-81, 2004.
[16] M. King Hubbert. Darcy's law and the field equations of the flow of underground fluids. Trans. AIME, 207(7):222$239,1956$.

[17] G.I. Barenblatt and A.A. Gilman. A mathematical model of non-equilibrium countercurrent capillary imbibition. Eng. Phys. Journal, 52(3):46-461, 1987.

[18] V.M. Ryzhik. On capillary imbibibtion by water of an oilsaturated reservoir. Soviet Academy Izvestia. Mechanics of Gas and Fluids, (2):149-151, 1960.

[19] D. B. Silin and T. W. Patzek. On Barenblatt's model of spontaneous countercurrent imbibition. Transport in Porous Media, 54(3):297-322, 2004.

[20] Zhi-An Luan. Some theoretical aspects of gravity drainage in naturally fractued reservoirs, SPE 28641. In 69th $S P E$ Annual Technical Conference and Exhibition, pages 357366, New Orleans, LA, 1994. SPE.

[21] G.I. Barenblatt, V.M. Entov, and V.M. Ryzhik. Theory of Fluid Flows through Natural Rocks. Kluwer Academic Publishers, Dordrecht, 1990.

[22] M. C. Leverett, W. B. Lewis, and M. E. True. Dimensionalmodel studies of oil-field behavior. Trans. AIME, 146:217$234,1943$.

[23] M. G. Crandall, L. C Evans, and P.-L. Lions. Some properties of viscosity solutions of Hamilton-Jacobi equations. Trans. Amer. Math. Soc. 282 (1984), 282:487-502, 1984.

[24] D. B. Silin. Generalizing Hopf and Lax-Oleinik formulae via conjugate integral. Monatshefte für Mathematik, 124:343-364, 1997.

[25] D. B. Silin. Viscosity solutions via unbounded set-valued integration. Nonlinear Analysis-Theory Methods 85 Applications, 31(1-2):55-90, 1998.

[26] James W. Johnson, John J. Nitao, Carl I. Steefel, and Kevin G. Knauss. Reactive transport modeling of geologic $\mathrm{CO}_{2}$ sequestration in saline aquifers: the influence of intraaquifer shales and the relative effectiveness of structural, solubility, and mineral trapping during prograde and retrograde sequestration. Technical report, Lawrence Livermore National Laboratory, 2002.

[27] W. H. Press, S. A. Teukolsky, W. T. Vetterling, and B. P. Flannery. Numerical Recipes in C: The Art of Scientific Computing. Cambridge University Press, Cambridge, 2nd edition, January 1993.

[28] M. Th. Van Genuchten. A closed-form equation for predicting the hydraulic conductivity of unsaturated soils. Soil Science Society of America Journal, 44(5):892-898, 1980.

[29] A.T. Corey. The interrelations between gas and oil relative permeabilities. Producers Monthly, 19:38-41, 1954. 